\title{
Optical Properties of Acupunctural Points as Diagnostic Method
}

\author{
B.R. Jovanić ${ }^{a, *}$, D. Nikolovski ${ }^{b}$, B. RadenkoviĆc And M. Despotovićc \\ ${ }^{a}$ Institute of Physics, CEP, Belgrade University, P.O. Box 68, 11080 Zemun, Serbia \\ ${ }^{b}$ Institute for Health, Pasterova 2, 26000 Pančevo, Serbia \\ ${ }^{c}$ FON, Lab. Simulation, Belgrade University, Jove Ilića 180, 11001 Belgrade, Serbia
}

\begin{abstract}
The goal of this work was to establish a connection between the optical parameters of the skin in which the acupuncture points have been settled and the pathological state of the organism. For the first time a connection between the optical characteristics of the specific skin parts (acupuncture points) and the state of the organism has been demonstrated. The optical parameter of the acupuncture point optical parameter (on surface of the reflectance curve between $470 \mathrm{~nm}$ and $660 \mathrm{~nm}$ ) conduct in accordance with the traditional Chinese medicine theories (balance disturbance within the organism, time change). Acquired results point to the possibility of using acupuncture point optical parameter measuring as a method to determine the number of therapeutical treatments during the traditional Chinese medical treatments.
\end{abstract}

PACS numbers: 87.64.--t

\section{Introduction}

The traditional Chinese medicine pays all the attention to maintenance of the balance between the antagonistic forces Yin-Yang. The principle is based on the following conclusions: (1) Yin-Yang is one of the main principle of Chinese medicine, according to which all physiological and pathological processes happen; (2) the disturbance of Yin-Yang balance is prerequisite for all illnesses within the organism; (3) by maintaining the Yin-Yang balance we carry out the treatment; (4) life energy always comes under the equilibrium of opposite forces law Yin-Yang; (5) illness is a result of disturbance of Yin-Yang balance.

Long ago, the Chinese found that the functional disorders of the interior organs or organic functions, both at human and animals, are almost always followed by painful sensations in certain places on the surface of skin (acupuncture points). Most acupuncture points have a diameter of $2-12 \mathrm{~mm}$, depending on the location in the body [1]. The points are always the same for a certain ill organ or organic function. In other words, sore points are the exterior manifestation of illness itself. Chinese doctors noticed that stimulation of these points (acupuncture points) had a favorable influence on the corresponding ill organ and thus turned each of these points into a therapy point. A sore point lasts as long as illness itself, and it vanishes once the balance has been reestablished. This obviously suggests that the metabolic processes, organs and complete condition of the organism can be influenced through the acupuncture points. An opposite correlation can also be assumed: that the acupuncture points may be influenced by the physiological processes,

\footnotetext{
* corresponding author; e-mail: brana@phy.bg.ac.rs
}

i.e. that any change within the organism (balance disturbance) would cause minor or bigger skin changes. Since the acupuncture points are placed in/on the skin, the mentioned changes would directly provoke the changes within the acupuncture points. This is not a question at issue, since the change of electrical resistance within the acupuncture points has been well examined, in dependence of the condition of the organism, and has been used diagnostically and therapeutically [2].

Several types of new diagnostic device that combine various diagnostic methods have recently appeared. The diagnostic methods are recognized either by the phenomenon that has been used as the system base, or by the scientist that introduced such a phenomenon first. Usually, the diagnostic procedure includes the measurement of various skin parameters [3] (electrical parameters, potential and current in the oral cavity) within the projection field of a certain biologically active point. The goal of these researches is to investigate optical (reflective) skin characteristics in the acupuncture points and to find out the correlation between the condition of an organism and reflection characteristics. But this method based on experimental facts that difference in the color between healthy and nonhealthy skin exists. The color of healthy skin is determined by the quantity of oxygenation of blood and presence or absence of the epidermal pigment [4].

\section{Materials and method}

Determination of the pathological state of the organism bases on two assumptions: (1) The acupuncture points are "special" spots in/on the skin and directed connect with pathological state of the organism; (2) Optical properties of the skin (the acupuncture points) are 
directly determined with pathological state of the organism. Mentioned assumptions based on literature data that: (a) pathological state of organism can cause change of skin reflection spectra and also area under the skin reflection spectra [4-6]; (b) the color of healthy skin is determined by the quantity of oxygenation of blood and presence or absence of the epidermal pigment (melanin, hemoglobin, oxyhemoglobin) [4] and (c) experimentally a fact is confirmed that the concentration of the mentioned pigments is defined by the physiological condition of the organism itself $[4,6]$. Now all procedure can be reduced on scanning the reflectance spectra of the acupuncture point and calculate the acupuncture point optical parameter (APOP) defined as area under the reflectance curve between $470 \mathrm{~nm}$ and $660 \mathrm{~nm}$. Every single reflection spectrum was normalized to reflection spectra of the $\mathrm{MgCO}_{3}$, which is usually accepted as standard $(100 \%)$. The detection unit was realized as an AVS-PC2000 (Avantes) with 2048-element linear CCD-array detector responsive from 200 to $1100 \mathrm{~nm}$. The spectrometer resolution was $0.2 \mathrm{~nm}$. The reflected light is collected and transmitted by the bifurcate detection fibers (400 mm diameter) which was coupled into AVS-PC2000. Excitation was realized with white LED diodes $(8 \mathrm{~mW})$ supplanting with constant current [7]. In our experiments the criterion of the limit condition is satisfied, as for any physical occurrences which depend on one stochastic argument [8]. At this way the precision was better that $5 \%$. On Fig. 1 experimental set-up is presented.

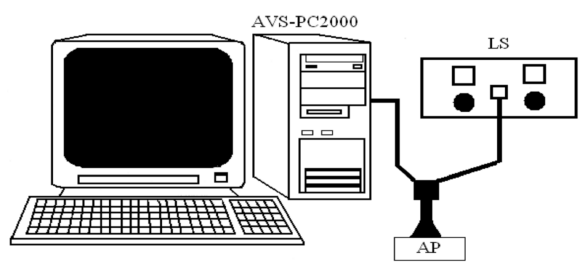

Fig. 1. Experimental setup. AVS-PC2000-spectrometer; LS - light source; AP - acupuncture point.

\section{Results and discussions}

At choice of "condition" of the organism, we had two criteria: (1) to be easily defined (easily noticeable symptoms) and (2) to be most suitable for laboratory measurements. The acupuncture points were chosen in accordance to old traditional Chinese medicine experience, taking into account their connection to the symptoms of the chosen condition of the organism [9].

We considered headache, most often reaction of students that are under stress condition before their exams. That is why we chose that condition of organism. The traditional Chinese medicine cures headache by treating the acupuncture point (Du.20) Baihui [10]. Since the tested students did not complain about any other discomfort, and stated that they were under stress, we concluded that stress directly caused headache. The con-

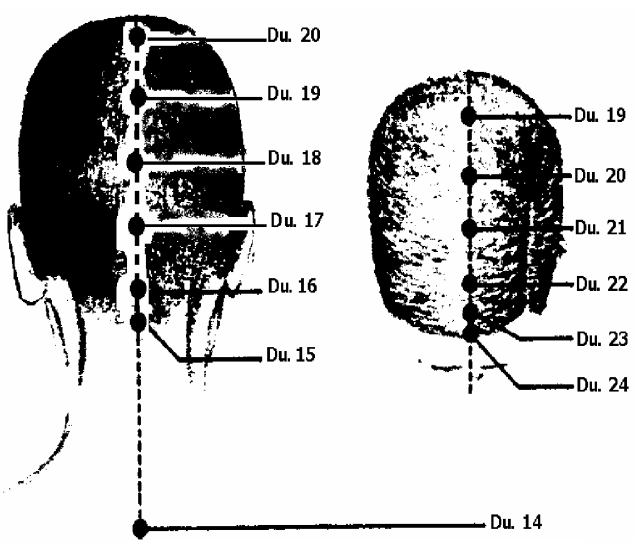

Fig. 2. Acupuncture point: Du.14, Du.15, Du.20.

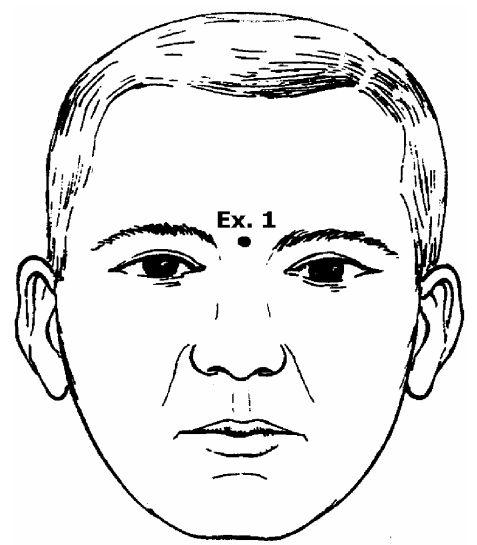

Fig. 3. Acupuncture point Ex.1.

trol group, without mentioned symptoms, counted 68 students, while there were 26 students suffering from headache in the stress group. The average APOP value for the acupuncture point (Du.20) for the control group, without headache, was $15120 \pm 320$ [rel.unit]. The average APOP value for the acupuncture point Du.20 for the stress group, with headache, was $28910 \pm 540$ [rel.unit]. Obviously, APOP value was almost twice higher (1.91) at the stress group in contrast to the control one. This gives us an idea that the increase of APOP value, in relation to the control group, may be index of stress.

Since plain cold accompanied with fever is often a condition of an organism with clearly noticeable and defined symptoms, we chose that condition as the one very suitable for research. The traditional Chinese medicine improves these symptoms by treating the acupuncture point (Li.4) Hegu [10] (Fig. 4/Li.4). The number of tested within the control group (healthy and without fever) was 42 , while there were 28 people with cold and fever in the other group. The APOP value for the acupuncture point (Li.4) was $55130 \pm 1200$ [rel.unit], while for those with cold and fever the APOP value for the acupuncture point (Li.4) was $126310 \pm 1450$ [rel.unit]. Like in the previous 
case, the condition of organism that is different from normal causes the increase of APOP. APOP value was 2.29 times higher at the stress group in contrast to the control one. In accordance with the previous discussion we may conclude that the increase of APOP is most probable in connection with the metabolic processes induced within the acupuncture point (Li.4) by the presence of virus in the organism and fever.

In the previous text we have mentioned the traditional Chinese medicine findings in the field of connection between physiologic processes within the organism and the acupuncture point.

In order to check the hypothesis on the influence of metabolic processes to APOP, we measured its value at people that were assumed to have had a considerable difference within physiological processes. The starting assumption was that the metabolic processes at people that did not eat meat (vegetarians) would be different than the ones at people that ate meat. Such a difference within the metabolic processes should have a different effect to APOP. We performed the measurements with five women of similar age (33-39 years). We chose three acupunctural points: (Du.14) Dazhui; (Du.15) Yemen and (Ex.1) Yintang. The points were selected without particular reason. The measured acupunctural point optical parameter APOP values are given in Table I.

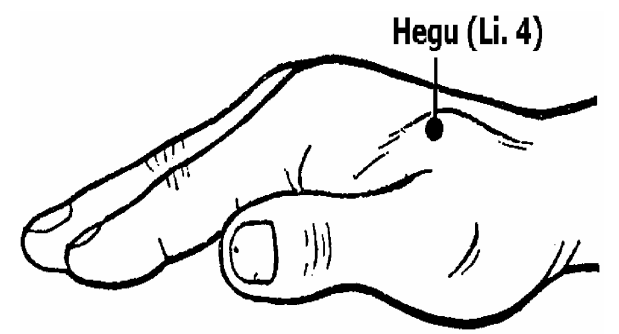

Fig. 4. Acupuncture point Li.4.

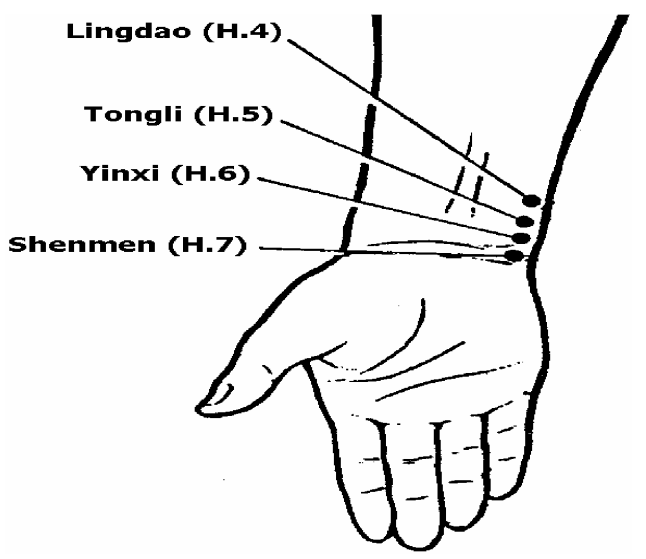

Fig. 5. Acupuncture point H.7.
TABLE I

$\mathrm{APOP}$ for vegetarians $\mathrm{APOP}_{\mathrm{V}}$ and nonvegeterians APOP $_{\mathrm{NV}}$ Fig. 2 (Du.14; Du.15); Fig. 3 (Ex.1).

\begin{tabular}{c|c|c|c}
\hline \hline & Du.14 & Du.15 & Ex.1 \\
\hline APOP $_{\mathrm{V}}$ [rel.unit] & $125580 \pm 812$ & $51540 \pm 220$ & $85280 \pm 922$ \\
APOP $_{\mathrm{NV}}$ [rel.unit] & $120660 \pm 542$ & $18090 \pm 155$ & $41210 \pm 623$
\end{tabular}

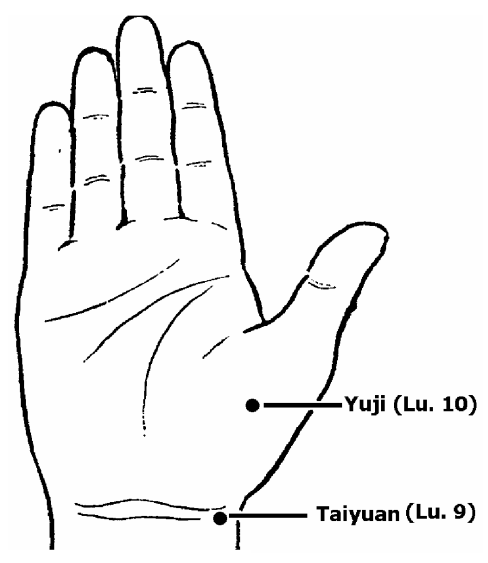

Fig. 6. Acupuncture point Taiyuan (Lu.9).

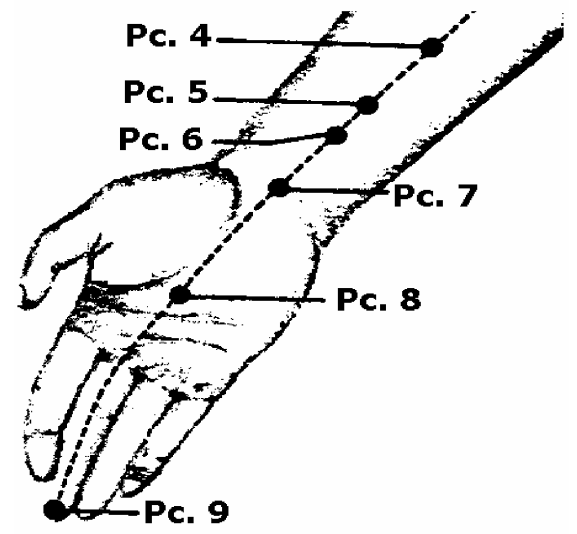

Fig. 7. Acupuncture point Daling (Pc.7).

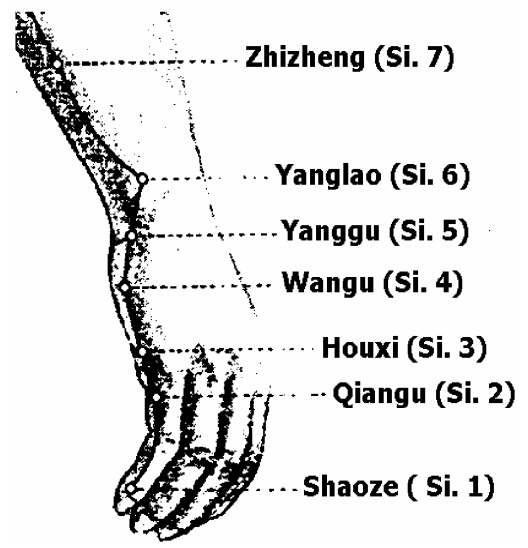

Fig. 8. Acupuncture point Si.4. 
TABLE II

APOP for different acupuncture point measuring at different time. Fig. 5 (H.7); Fig. 6 (Lu.9); Fig. 7 (Pc.7); Fig. 8 (Si.4)

\begin{tabular}{c|c|c|c}
\hline \hline APOP [rel.unit] & $0[\mathrm{~min}]$ & $60[\mathrm{~min}]$ & $120[\mathrm{~min}]$ \\
\hline Lu.9 & $61750 \pm 2100$ & $48970 \pm 1050$ & $44200 \pm 3000$ \\
Pc.7 & $83120 \pm 1820$ & $74540 \pm 1110$ & $72690 \pm 2120$ \\
Si.4 & $72880 \pm 1180$ & $74870 \pm 3300$ & $60400 \pm 1100$ \\
H.7 & $88290 \pm 2190$ & $93160 \pm 1480$ & $96690 \pm 2300$
\end{tabular}

Analyzing the results from Table I, we noticed a considerable difference in skin reflection, i.e. APOP within the two acupuncture points Du.15 and Ex.1. There was no obtained difference in APOP in one acupuncture points (Du.14) between vegetarian and nonvegeterian persons. In other two considered acupuncture points Du.15 and Ex.1, a difference between APOP for vegetarian and nonvegeterian persons was obtained. For vegetarian persons APOP for Du.15 acupuncture point was almost three times (2.85) higher than for nonvegeterian persons. For vegetarian persons APOP for Ex.1 acupuncture point was 2.07 higher than for nonvegeterian persons. Those made us conclude that our assumption regarding nutrition habits, i.e. the ATP metabolism, was correct. A detailed analysis of causes of skin reflection changes within ATP, i.e. APOP, requires further research.

It has been written that life energy in the acupuncture points changes within the $24 \mathrm{~h}$ course, so that it reaches its $2 \mathrm{~h}$ peak at certain time and drops to its minimum $12 \mathrm{~h}$ later in the same acupuncture point [9, 11]. Life energy within the acupuncture points alters naturally within $24 \mathrm{~h}$ course. Beginning from some level it starts to grow and reaches its $2 \mathrm{~h}$ maximum, then decreases, to reach its minimum $12 \mathrm{~h}$ later at the same canal. The energy change periodicity within the acupuncture points causes periodicity changes within electrical resistance of the acupuncture points [12]. In accordance with aforementioned, one might expect that APOP changes in accordance to the energy changes within the acupuncture points, should the measurement be performed for more than $2 \mathrm{~h}$. Should the time at which the energy within the acupuncture points is neither on maximum nor on minimum is chosen, APOP would either tend to decrease or to increase. We performed measurements at eleven 37 old male. The chosen points were: (H.7) Shenmen heart canal point, (Lu.9) Taiyuan - lungs canal point, (Pc.7) Daling - pericardium canal point, (Si.4) Wangu - small intestine canal point. The maximum within the mentioned points is reached [6]: (H.7): 11-13 h; (Lu.9): 3-5 h; (Pc.7): 19-21 h; (Si.4): 13-15 h.

The measurement results are given in Table II. Table shows that APOP within all acupuncture points changes with time. At three acupuncture points (Lu.9), (Pc.7) and (Si.4) we notice a decreasing trend of APOP, which indicates that in these acupuncture points the energy tends to its daily minimum. At (H.7) we notice an in- creasing trend of APOP, which indicates that the energy tends to its daily maximum.

In accordance with the research described in this work, we can say that any deviation from the balance would result with changes of optical characteristics of the acupuncture points. However, recognizing the APOP values for balanced condition (healthy organism), the number of therapies (stimulations of acupuncture points) would be determined according to its value. The therapy would be performed as long as we reach the APOP value of a healthy and well-balanced organism.

\section{Conclusion}

For the first time it has been demonstrated that there is a connection between the optical characteristics of specific skin parts (acupuncture points) and condition of an organism. It has also been demonstrated that there is a significant change of optical activities of the acupuncture points, depending on time. The optical parameter APOP of acupuncture points behaves in accordance with the theories of the traditional Chinese medicine (change and disturbance of balance of the organism, time change, left/right effect).

\section{Acknowledgments}

This work was supported by grant No. 141007 from MNRS.

\section{References}

[1] Y. Omura, Jap. Acupuncture J., Japan. Med. 692, 129 (2001).

[2] G.F. Portnov, Electropuncture Reflexotherapeutics, Zinatne, Riga 1982 (in Russian).

[3] D. Raković, Int J. Acupuncture, Electro-Therapeutics Res. 16, 88 (1991).

[4] J.W. Feather, D.J. Ellis, G. Leslie, Phys. Med. Biol. 33, 711 (1988).

[5] E. Angelopoulou, http://repository.upenn.edu/cis reports/584 (1999)

[6] V.P. Wallace, D.C. Crawford, P.S. Mortimer, R.J. Ott, J.C. Bamber, Phys. Med. Biol. 45, 735 (2000).

[7] http://www.theledlight.com/pdf/15DEGWH.pdf .

[8] F. Bardou, J.P. Bouchaud, A. Aspect, C. Cohen-Tannoudji, Levy Statistics and Laser Cooling, Cambridge Univ. Press, UK 2002.

[9] Y. Omura, Acupuncture Medicine: Its Historical and Clinical Background, Japan Publ. Inc., Tokyo 1982.

[10] P. Marić, Basics Chinese Acupuncture Naučna knjiga, Beograd 1989, p. 110 (in Serbian).

[11] F. Mann, Acupuncture: Cure of Many Diseases, William Heinemann Medical Books Ltd, London and Sydney 1971, p. 61.

[12] Z. Jovanović-Ignjatić, D. Raković, Int. J. Acupuncture Electro-Therapeutics Res. 24, 105 (1999). 